\title{
Modalidades en docencia universitaria
}

\author{
M. Ed. Marcelo Arroyo Jiménez
}

La educación es una manifestación esencialmente social. Esta extensión social aparece tanto en la naturaleza misma del proceso educativo como en los contenidos, hábitos y valores que se transfieren en la acción educativa. Por consiguiente, la educación es siempre consecuencia del momento histórico en que sucede, del lugar donde se realiza, de la cultura dominante, así como de la estructura sociopolítica vigente (Sarramona, 1994: 11).

La materialización de las respuestas que la sociedad actual demanda del sistema educativo se encuentra con limitaciones, intrínsecas y extrínsecas al mismo sistema, que han de ser tenidas en cuenta para actuar sobre ellas y aminorarlas en lo posible, si no suprimirlas totalmente. Entre las limitaciones que afectan todos los niveles educativos de nuestro país tenemos:

1. Preparación de los docentes.

2. Financiamiento / elevado costo de la educación.

3. Lentitud en los cambios pedagógicos / reformas educativas. 
4. Condicionamiento del medio sobre las posibilidades y resultados del sistema educativo.

La educación superior ha cambiado hondamente, y los que están verdaderamente comprometidos con la labor académica deben todavía resolver las secuelas de estos cambios. Los sistemas y las instituciones académicas han afrontado presiones ante un número creciente de estudiantes y cambios demográficos, demandas de índole contable, reconsideración del rol social y económico de la educación superior, consecuencias de los cambios socio-políticos y el impacto de las nuevas tecnologías, entre otras cosas. Mientras que los programas universitarios funcionan en un ámbito nacional, los desafíos se presentan en una escala global, lo cual hace que los centros de formación superior busquen solventar sus necesidades académicas de manera que respondan a las expectativas y satisfagan la demanda educativa en auge (Altbach y McGill Peterson, 2000: 21).

Ante esta perspectiva, la docencia universitaria se vale, básicamente, de dos tipos de modalidades para cumplir su propósito, a saber: docencia presencial y docencia a distancia. A continuación se presentarán los conceptos y características primordiales de cada modalidad, así como un análisis de ambas a la luz de sus fortalezas, debilidades, tendencias actuales y condiciones de logro.

\section{MODALIDAD PRESENCIAL}

Tradicionalmente, la educación se da dentro de un espacio físico (las aulas), por medio del proceso de enseñanza-aprendizaje que se facilita entre un docente que ofrece un curso a los alumnos, en el mismo espacio y tiempo, y éstos reciben los conocimientos que se les transmiten, por medio de la comunicación verbal, expresión corporal, los 
medios visuales y sonoros y aquellos medios didácticos de apoyo que ayudan a este tipo de enseñanza. Ejemplos de este tipo de modalidad son conferencias, seminarios, clases, etc.

A esta modalidad de enseñanza se le ha llamado educación presencial o formal, resaltándose que el proceso de enseñanza debe estar encuadrado dentro de un mismo tiempo y espacio (el aquí y el ahora), en donde se presentan al mismo tiempo el maestro y los alumnos, bajo una atmósfera académica presencial y con métodos tradicionales de transmisión del conocimiento. Esto se aplica en nuestro sistema educativo por igual en todos los ciclos de la Educación General Básica y la Educación Diversificada, así como la Educación Superior, incluyendo los Postgrados.

Los cimientos de la educación presencial son: las aulas, el diálogo como esquema de enseñanza, la transmisión del conocimiento de manera oral y la relación en el mismo espacio-tiempo del maestro-alumno. Este tipo de modalidad abarca, además de las anteriores relaciones, todos aquellos apoyos didácticos, las dinámicas de grupo, las técnicas de enseñanza y la educación escolarizada, así como otras muchas características de la enseñanza-aprendizaje tradicionales.

\section{MODALIDAD A DISTANCIA}

En el siglo pasado, en la década de los sesenta, las sociedades occidentales vivieron una profunda transformación que, en el campo de la educación superior, se reflejó en la exigencia de elevar la calidad de los estudios de manera paralela a la flexibilización de la administración escolar.

Lo anterior impulsó la adecuación de la adquisición de conocimientos a las necesidades y perspectivas individuales de la población. Por ello, resultaba urgente contar 
con opciones adicionales a la educación escolarizada o presencial, para así poder cursar estudios superiores con estructuras académicas más flexibles. De esta manera nació la educación abierta o a distancia.

Considerada como pionera en el diseño y aplicación sistemática de modelos innovadores de educación abierta, en 1967 se creó la Open University en Inglaterra, la cual inició el desarrollo de un sistema de enseñanza abierta con mecanismos flexibles y con sus propios títulos y grados. A partir de este momento histórico, surgen muchos programas a distancia en el mundo, buscando abrir las posibilidades de acceso a educación.

\subsection{Definición de Educación a Distancia}

La Educación a Distancia es una representación genérica, difícil de definir, debido a que en ella se incluyen las estrategias de enseñanza-aprendizaje, los medios de difusión masivos, la transmisión de conocimientos por algún medio de comunicación y las expectativas del maestro y el alumno dispersos y alejados, sin ningún contacto físico inmediato entre sí, salvo los medios de comunicación. En el mundo a este tipo de enseñanza se le ha denominado de diferentes formas: educación abierta, educación a distancia, educación virtual.

La Enseñanza a Distancia es un proceso de enseñanza-aprendizaje que se vale de la utilización de diversos medios de comunicación social como correo, prensa, radio, televisión, teléfonos, cassettes, computadoras, etc., con alumnos disgregados y alejados entre sí.

También, es preciso aclarar que mediante la modalidad a distancia es posible el desarrollo de diversos conocimientos, pero también de habilidades y actitudes, el empleo de variados recursos didácticos -señalados 
anteriormente, permiten el favorecimiento de diversas capacidades en los individuos. Por ello es que dicha modalidad puede ser utilizada tanto al inicio de los programas de capacitación, como en los programas de actualización y especialización. (Alvarado Céspedes y otros, 1999: 75)

Entre las razones que propician la educación a distancia tenemos:

- Sociales, que permiten una superación cultural a muchas personas que no pueden asistir regularmente a un centro educativo por motivos laborales, enfermedades, estancia en el extranjero, etc.

- Técnicas, gracias a que los avances tecnológicos al servicio de la educación permiten una comunicación eficaz, rápida y masiva.

- Económicas, que permiten una disminución de los altos costos a que se ve sometida hoy la educación.

Las características que se encuentran en este tipo de modalidad de enseñanza son:

- Pretende ofrecer educación en forma masiva.

- Está dirigida especialmente a población adulta.

- La interrelación no presencial entre el docente y el alumno.

- La enseñanza y el aprendizaje ocurren de manera separada.

- Se superan las limitaciones de tiempo y espacio; sin embargo, se debe asistir a la realización de exámenes, entrega de tareas, matrícula, etc.

- La comunicación se realiza a través una interacción no física, que se da por medio de algún medio de comunicación: escrito, audiovisual, electrónico, multimedia, etc. 
- El docente actúa como tutor y guía del aprendizaje, aunque se programen sesiones de tutorías.

- El alumno, desde un lugar alejado, desarrolla sus actividades de aprendizaje y las reporta al maestro (autoaprendizaje).

- La institución educativa ofrece apoyo a nivel central y en los centros regionales.

(Alvarado Céspedes y otros, 1999: 77)

La educación presencial es distinta a la educación a distancia. Sus diferencias se ven reflejadas en el cuadro $\mathrm{N}^{\circ} 1$.

Flechsig y Schiefelbein (1985) exponen tres principios didácticos identificados con la modalidad de estudio a distancia, mismos que refuerzan la teoría expuesta en las páginas precedentes:

a) Aprendizaje mediante trabajo individual, el alumno recurre generalmente a la información e indicaciones para el aprendizaje recibidas en los materiales de aprendizaje y estudia sin apoyo de un profesor o compañeros.

b) Aprendizaje con medios, esto significa que el aprendizaje sólo depende de la información transmitida (desde la central), sin que existan otras experiencias reales que determinen el proceso de aprendizaje.

c) Aprendizaje mediante tareas (aprender realizando tareas), el alumno aprecia el éxito de su aprendizaje por la corrección y comentarios de las tareas que realiza (solicitadas en los materiales).

Además, Flechsig y Schiefelbein explican que la forma correcta de aplicar este modelo de enseñanza se basa en cuatro fases, que van desde la oferta del aprendizaje, es 


\section{CUADRO N${ }^{\mathrm{O}} 1$}

Diferencias entre Educación Presencial y Educación a

Distancia

\begin{tabular}{|c|c|c|}
\hline & EDUCACIÓN PRESENCIAL & EDUCACIÓN A DISTANCIA \\
\hline 1 & $\begin{array}{l}\text { El profesor y los estudiantes están } \\
\text { físicamente presentes en un mismo } \\
\text { espacio-tiempo (durante las clases). }\end{array}$ & $\begin{array}{l}\text { El profesor y los estudiantes pueden no } \\
\text { estar presentes físicamente en el mismo } \\
\text { espacio ni en el mismo tiempo. }\end{array}$ \\
\hline 2 & $\begin{array}{l}\text { La comunicación es directa entre } \\
\text { profesor y los estudiantes y se da en } \\
\text { el mismo espacio-tiempo, sin nece- } \\
\text { sidad de usar elementos adicionales. }\end{array}$ & $\begin{array}{l}\text { Para que la comunicación se produz- } \\
\text { ca, es necesario contar con elemen- } \\
\text { tos mediadores entre el docente y } \\
\text { alumnos (ambos no presentes). }\end{array}$ \\
\hline 3 & $\begin{array}{l}\text { La voz del profesor y su expresión } \\
\text { corporal son los medios de comu- } \\
\text { nicación por excelencia. Se les lla- } \\
\text { ma presenciales a estos medios por- } \\
\text { que restringen la comunicación a } \\
\text { un aquí y a un ahora. }\end{array}$ & $\begin{array}{l}\text { La voz y el esquema temporal, o son } \\
\text { sustituidos por otros medios no-pre- } \\
\text { senciales, o serán registrados en gra- } \\
\text { baciones sonoras y visuales para ser } \\
\text { transmitidos luego a otro espacio y } \\
\text { en otro tiempo. }\end{array}$ \\
\hline 14 & $\begin{array}{l}\text { Los medios visuales y sonoros son } \\
\text { poco utilizados en la clase conven- } \\
\text { cional y sólo sirven como apoyos } \\
\text { didácticos o para complementar la } \\
\text { acción del profesor. }\end{array}$ & $\begin{array}{l}\text { Los medios visuales, sonoros y otros, } \\
\text { no son simples ayudas didácticas, } \\
\text { sino son los portadores de conoci- } \\
\text { miento que sustituyen al profesor. }\end{array}$ \\
\hline 5 & $\begin{array}{l}\text { La comunicación oral, característi- } \\
\text { ca en la enseñanza convencional, } \\
\text { está acompañada normalmente por } \\
\text { gestos y movimientos de la comu- } \\
\text { nicación no verbal. }\end{array}$ & $\begin{array}{l}\text { Adquieren gran importancia los } \\
\text { medios como la palabra escrita (do- } \\
\text { minante por excelencia), además de } \\
\text { la radio, la televisión y otros medios } \\
\text { audiovisuales. }\end{array}$ \\
\hline 6 & $\begin{array}{l}\text { La relación directa, presencial, de } \\
\text { los que se comunican hace que el } \\
\text { diálogo pueda producirse también } \\
\text { aquí y ahora, de manera inmediata. }\end{array}$ & $\begin{array}{l}\text { La relación no-presencial de los que } \\
\text { se comunican, es una forma de diá- } \\
\text { logo que por no acontecer aquí ni } \\
\text { ahora, bien puede llamarse "diálogo } \\
\text { diferido". Es decir, el comunicador } \\
\text { debe emitir un mensaje completo y } \\
\text { esperar un tiempo para recibir la co- } \\
\text { municación de retorno en forma si- } \\
\text { milar, como por ejemplo una carta. }\end{array}$ \\
\hline
\end{tabular}

Fuente : Muñoz Razo 
decir, se ponen en evidencia las posibilidades e intereses del alumno, pasando luego por la recepción de la información, la interacción propia de estudio y solución de tareas hasta concluir con la corrección y comentarios de los trabajos realizados, para prepararse de nuevo para nuevas tareas de aprendizaje.

\subsection{Educación virtual}

La educación virtual es un sistema y modalidad educativa que surge de la necesidad propia de la educación y la tecnología educativa. La UNESCO (1998) la define como «entornos de aprendizajes que constituyen una forma totalmente nueva, en relación con la tecnología educativa... un programa informático - interactivo de carácter pedagógico que posee una capacidad de comunicación integrada. Es una innovación relativamente reciente y fruto de la convergencia de las tecnologías informáticas y de telecomunicaciones que se ha intensificado durante los últimos diez años".

La educación virtual como concepto ha generado bastantes discusiones en el marco social educativo. Muchos la han catalogado una enseñanza bajo fantasías, descontextualizada, mientras que otros arguyen que la formación se da por medio de Internet. Debido a lo anterior, se plantean las siguientes interrogantes: ¿Qué es lo virtual? ¿Existe verdaderamente una educación virtual? ¿Se ha de convertir la educación formal o alternativa en educación virtual? ¿Dónde quedarán las aulas? ¿Cómo desafía la educación virtual a los docentes?

A partir de estos cuestionamientos, se aduce que la educación virtual puede entenderse diferente a la educación a distancia, puesto que algunos teóricos tratan estos conceptos como sinónimos, no teniendo presente que en 
su esencia difieren en sus procesos (Tintaya). Además, se destaca que la educación virtual, como la educación del siglo XXI, tiene los siguientes principios:

\section{- La auto-educación \\ - La auto-formación \\ - La desterriorialización \\ - La descentralización \\ - La virtualización \\ - La tecnologización \\ - La sociabilidad virtual}

No se omite mencionar que el tipo de enseñanza que se puede brindar por medio de la Red está determinado por particulares tales como su estructura asociativa, no-lineal, y jerárquica, su capacidad de anexar diversos medios, y su poder de comunicación sincrónica o asincrónica, las cuales hacen de este medio un escenario educativo muy poderoso y singular. Desde una perspectiva constructivista se pueden ver estos atributos de la Red como instrumentos para que los alumnos construyan conocimiento en forma colaborativa y consigan una mejor comprensión de los conceptos (Miller y Miller: 2000, citados por Henao Álvarez: 2004).

La organización de la Red no está circunscrita a enlaces entre documentos en formato textual, sino que incluye otros medios como dibujos, fotografías, animaciones, videos y sonidos. El término hipermedios expresa la naturaleza multimedial e hipertextual de la información disponible en la Red. Utilizando diversos recursos se pueden representar con gran precisión fenómenos, situaciones y contextos que constituyen ambientes de aprendizaje auténticos.

La mayoría de los centros educativos que ofrecen educación virtual utilizan la Internet y sus herramientas para permitirles a los estudiantes, a través de una página Web 
creada por ellos, acceder a un campus virtual que, básicamente, consiste en una aplicación telemática que posibilita la interrelación entre los distintos miembros de una universidad. De acuerdo con Henao Álvarez (2004), dentro de este campus virtual está enmarcada el aula virtual, que es el sitio donde se encuentra información específica sobre el curso o cátedra, Chat, foros de discusión, correo electrónico para intercambiar información con el docente y compañeros, entre otras cosas.

Existen múltiples programas para ofrecer a los alumnos entornos virtuales adecuados a sus exigencias de conocimiento a través de Internet, que facilitan el intercambio de información entre estudiantes y docentes. Algunos de los programas, según Henao Álvarez, son:

- WebCT: Este servidor puede albergar un gran número de cursos. El programa se puede bajar desde Internet en forma gratuita, la institución prepara los cursos y sólo cuando se empieza a usar el programa con alumnos matriculados es necesario conseguir las licencias correspondientes.

- TopClass: Es un software para servidores diseñado para desarrollar formación a través de Internet. Se puede acceder a este desde múltiples plataformas utilizando navegadores estándares. Además, proporciona a los estudiantes un ambiente de clase virtual con soporte para la construcción de mensajes y la discusión.

- Learning Space: Es un software de IBM lotus, conocido como e-learning o educación en línea, que incorpora la fortuna del aprendizaje de grupo con la elasticidad del aprendizaje individual. Learning space (Espacio de aprendizaje) está compuesto por módulos interconectados, cada uno de los cuales es una base de datos en Lotus notes, que consta de: programación 
del curso, centro de medios, cuarto de colaboración, administración de evaluaciones y perfiles de la clase.

- Virtual-U: Es una plataforma de enseñanza - aprendizaje distribuido en línea, sencilla tanto para el maestro como para el alumno, que permite enfocarse en el modelo de aprendizaje y en el diseño de instrucción de los cursos.

\section{DOS CASOS: MODALIDAD A DISTANCIA Y MIXTA}

\section{UNED: MODELO DE EDUCACIÓN A DISTANCIA EN COSTA RICA}

La Universidad Estatal a Distancia (UNED) es una institución de carácter público que goza, desde su fundación en 1977, de autonomía. Su misión es ofrecer educación superior a todos los sectores de la población, especialmente a aquellos que por razones económicas, sociales, geográficas, culturales, de discapacidad o de género, requieren oportunidades para una inserción real y equitativa en la sociedad. (UNED 2005, Web)

Para ello hace uso de los diversos recursos pedagógicos, tecnológicos y otros medios de comunicación social que permiten la interactividad, el aprendizaje independiente y una formación humanista, crítica, creativa y de compromiso con la sociedad y el medio ambiente.

La UNED tiene como compromiso la excelencia académica, el desarrollo de la cultura, la ciencia, el arte y los derechos humanos para la construcción de una sociedad justa y una cultura de paz. (UNED 2005, Web)

Ramírez Sánchez (2005), docente de este centro de educación superior desde hace más de veinte años, indica que la educación a distancia es un buen sistema de enseñanza - aprendizaje para democratizar la educación de un 
país. Manifiesta que esta modalidad usa diversos recursos para llevar a cabo la labor docente, entre los que se encuentran las unidades didácticas modulares, mismas que contienen un conjunto integrado de medios escritos, programas audiovisuales, programas de radio, guías de estudio, discos compactos, comunicaciones por correo electrónico, tutorías y otros. Al mismo tiempo, para Ramírez Sánchez es importante destacar que en la UNED se atiende también población específica: con discapacidad y privados de libertad, a quienes se les brinda apoyo especial. Además, se ofrece un programa de estudiantes en el extranjero y hay 30 estudiantes en otros países que estudian a distancia.

Según Ramírez Sánchez, la modalidad a distancia que en la actualidad lleva a cabo la UNED de Costa Rica, se caracteriza por ser una modalidad individualizada y autosuficiente, apoyada fundamentalmente con las tutorías en cada materia (estas tutorías son impartidas cada quince días por espacios de dos horas cada sesión). Estas reuniones se desarrollan en uno de los 31 centros universitarios ubicados en todo el territorio nacional.

\section{DIVISIÓN DE EDUCACIÓN RURAL, CIDE: MO. DELO MIXTO}

La Universidad Nacional, con el compromiso de dar respuestas a las necesidades de los sectores populares, heredó de la Escuela Normal ${ }^{2}$ las demandas de la educación rural del país. Así, el Centro de Investigación y Docencia en Educación (CIDE) y la División de Educación Rural (DER), asumieron la responsabilidad de formar docentes que ejercen

\footnotetext{
2 La Escuela Normal, fundada en 1914, se constituyó como un movimiento pedagógico innovador, con marcado contenido nacionalista. Su finalidad, inminentemente social, era el desarrollo educativo, a través de la formación de maestros que pudieran servir a todo el país. (Dengo, 2004)
} 
su labor en zonas rurales del país. Esta labor se vinculó con una modalidad mixta, es decir, la modalidad presencial y a distancia.

Inicialmente, se atendía a la población de maestros en servicio sin título docente, pues la modalidad presencial y a distancia nació para atender a los estudiantes de zonas alejadas de las sedes universitarias. Uno de los aportes más significativos de la DER es el haber legitimado, a nivel de educación superior, un proceso de formación de educadores utilizando una modalidad mixta. (DER, Plan de Estudios, 2005)

En la actualidad, luego del rediseño curricular de la carrera, se prosigue con los principios que fundamentaron las acciones académicas del CIDE y de la DER, pero la población meta estudiantil se redimensiona, lo que permite la inclusión de estudiantes con aprobación del Bachillerato de Educación Diversificada, provenientes de zonas rurales.

Para la DER, la modalidad presencial y a distancia se entiende como "un proceso de adquisición del conocimiento que se da en dos ambientes de aprendizaje, interrelacionados, caracterizados por el énfasis de las estrategias metodológicas empleadas en cada una de ellos. Así, se permite la construcción del conocimiento basado en la comunicación, la reflexión grupal y el trabajo individual. La investigación, la evaluación y la producción sobre las experiencias, constituyen un proceso de aprendizaje con significado." (DER, Plan de Estudios, 2005)

En esta modalidad, la clase presencial (cada quince días se imparten dos materias del bloque del semestre) pretende problematizar el objeto de estudio, analizarlo y sistematizarlo. Para lograr esta meta, las sesiones buscan crear un ambiente de reflexión, y contemplan la comprobación individual, la profundización de los temas y la asignación del trabajo a distancia. Todo esto ocurre a través de un proceso 
acción-reflexión-acción. Por otra parte, la modalidad a distancia se considera el trabajo fuera del aula universitaria. Este proceso promueve la auto-capacitación, abarca lectura y análisis de documentos, reflexión del trabajo realizado, realización de experiencias con grupos de niños y niñas en relación con estrategias analizadas en clase, confrontación de la teoría con la práctica y elaboración de informes. De esta manera, el estudiante integra conocimientos adquiridos en la clase (presencialmente) y los confronta con la práctica.

Con respecto a los recursos metodológicos empleados por la DER para impartir los cursos, Cerdas Rivera (2005) -Sub-directora de la División de Educación Ruralindica que se utilizan guías de trabajo y material de lectura, así como trabajo en línea (este recurso se utiliza especialmente para realizar consultas o tutorías, no como un portal abierto de material). También, se efectúan giras de estudio a zonas rurales e investigaciones de campo con instrumentos de cotejo, con el propósito de que los estudiantes estén en contacto directo con el objeto de estudio.

No se omite mencionar que la modalidad de la DER está administrada desde el Campus Omar Dengo, pero hay interés en las otras sedes regionales (Chorotega y Brunca) de iniciar el proceso administrativo y asumir la responsabilidad directa de conformar sus propias escuelas para llegar a la población rural de las regiones.

\section{ANÁLISIS SOBRE LAS MODALIDADES EN DOCENCIA}

Las modalidades en docencia universitaria, como bien se puede inferir de su perspectiva teórica y su aplicación basándonos en los dos casos expuestos anteriormente, no son completamente excluyentes, más bien parece que la 
tendencia actual es tratar de integrarlas, de manera que se aprovechen sus fortalezas y se minimicen sus debilidades. De esta forma, los docentes pueden aproximarse al objeto de estudio de la educación superior desde diversos ángulos, valiéndose de las herramientas que las modalidades ponen a su alcance. A continuación, se enuncian una serie de fortalezas, debilidades, tendencias actuales y condiciones de logro, que se desprenden de la presentación teórica de cada modalidad, así como del estudio de los dos casos mencionados.

\subsection{Fortalezas}

La Educación Presencial presenta una serie de fortalezas sobre todo en la interrelación profesor - estudiantes, puesto que ambos comparten en el salón de clases perspectivas de análisis sobre uno o varios temas, hacen valoraciones, se interrogan mutuamente $y$, de este compartir, se re/ construyen (nuevos) conocimientos. Esta confrontación de ideas es inmediata, así como la comunicación de lineamientos que guían el proceso de aprendizaje, lo cual produce que se puedan evacuar dudas o preguntas en el momento. A la vez, se evidencia que el contacto personalizado de profesor - grupo da más sentido de pertenencia a una institución o plan de estudios.

Por su parte, la Educación a Distancia presenta como fortaleza indiscutible la posibilidad real de atención masiva, porque el cupo no es un factor limitante. Es decir, que la cobertura a nivel nacional ( 31 centros educativos en el caso de la UNED), aunado a la flexibilidad de horarios y los bajos costos, constituyen una motivación para que cada estudiante satisfaga necesidades de formación superior cuando no pueden ir a clases regulares en las universidades debido al trabajo, tiempo, dinero o espacio, y que continúen sus estudios sin dejar de trabajar y recibir un salario. Además, 
esta modalidad hace accesible la educación y capacitación a estudiantes en áreas apartadas. Otra fortaleza se vislumbra en el área docente, pues los centros y programas de estudio poseen y producen textos escritos por expertos. Esto, a la vez, promueve la auto-educación, es decir, que cada estudiante es el responsable directo de su proceso educativo, y el aprendiente se puede visualizar como el administrador de su aprendizaje.

Más fortalezas de la modalidad a distancia son: rompe con las barreras de tiempo y espacio; es innovadora, debido a que el aprendizaje es multidireccional y se utilizan diferentes recursos metodológicos y tecnológicos para lograr los objetivos educativos. En el plano evaluativo, los estudiantes se ven más involucrados y activos en su proceso, esto hace que la formación tenga más características andragógicas: se promueve, por ejemplo, la autorreflexión del proceso de formación por medio de la auto-evaluación.

\subsection{Debilidades}

Con respecto a las debilidades, en la Educación Presencial el centro de acción / reflexión es el docente, y se asume que éste posee todo o la mayoría del conocimiento y que es su obligación transmitir el conocimiento. Este condicionamiento hace que en muchos casos los estudiantes se vean como entes pasivos, y que la responsabilidad del aprendizaje esté en el docente, lo cual la convierte en una educación totalmente vertical. Además, el profesor usa tradicionalmente la metodología expositiva, por lo que en muchos casos la lección se torna monótona y poco participativa. Con respecto a la evaluación, la modalidad presencial se caracteriza por ser unidireccional y no permite que ocurran procesos democráticos donde docentes y estudiantes discutan la metodología evaluativa. Una debilidad de 
esta modalidad es que no puede abarcar grandes cantidades de personas, debido al carácter presencial, lo cual la convierte en restringida a ciertos grupos de población que habitan cerca del campo educativo.

Referente a la Educación a Distancia, se puede decir que los factores económicos pesan como una debilidad en esta modalidad. Lo anterior se debe a que la tutoría, metodología común de ésta, se torna en gastos sumamente caros para el sistema educativo cuando ésta debe ser personalizada. Además, se sacrifica la interacción social por el trato individual. En este contexto, el profesor se limita en las tutorías a evacuar dudas y no existe un verdadero intercambio o confrontación de ideas, pues la comunicación se limita a clarificar lo que dicen los textos.

Otra debilidad de la modalidad a distancia es la deserción en estudiantes que no se adaptan al método de estudio, debido a la falta de capacitación en estrategias y métodos de estudio a distancia. Además, este tipo de modalidad se asocia con estudio aislado o solitario, especialmente cuando se presupone que todo el proceso educativo está mediado por libros y recursos tecnológicos. El estudiante, desde esta perspectiva, se convierte en un sujeto que carece de acceso a otros individuos para compartir lo que aprende en su proceso, situación que es muy cuestionada por docentes que consideran que el contacto e interacción son vitales en los procesos de formación humana.

Además, la educación virtual plantea un gran desafío en el plano evaluativo, pues los docentes requieren evaluar no sólo el rendimiento académico, sino también otras variables emergentes que son extremadamente difíciles de medir, por ejemplo, el aprendizaje emocional de los estudiantes, el conocimiento previo sobre la temática, identificación del estudiante con la comunidad educativa, etc. 


\subsection{Tendencias actuales y condiciones de logro}

Con respecto a las tendencias actuales, las modalidades en docencia universitaria evidencian una complementariedad, no tanto en la concepción teórica como en el ejercicio docente. Esta conjunción se manifiesta producto de factores como la descentralización de los servicios, la apertura y fortalecimiento de los centros universitarios, la inmersión y participación en las comunidades en las que están ubicados los centros universitarios -especialmente las sedes que no se encuentran en el Gran Área Metropolitana-, y la actualización, diversificación y mejoras en los equipos tecnológicos.

Además, la línea divisoria entre la modalidad presencial y a distancia cada vez es más delgada, pues ambas modalidades hacen uso de los recursos metodológicos de la otra con fines académicos. Como se evidencia en el caso de la DER, el plan de estudios contempla explícitamente una modalidad mixta, en la que se llevan a cabo sesiones presenciales, pero también se implementa la modalidad a distancia, con el fin de que los estudiantes puedan, en su proceso de formación, apropiarse de las vivencias que implica la educación rural.

Al diseñar procesos y estructuras organizativas, la educación presencial y a distancia se irán integrando sistemáticamente dentro de un ámbito curricular e institucional, lo cual podría incrementar los niveles de calidad de las prácticas asociadas. Además, se evidencia un gran interés por la Educación a Distancia, porque se plantea todo un desafío en el mundo por conocer más sobre esta modalidad, debido a la multiplicidad de opciones que presenta.

Asimismo, hay un creciente interés en abrir programas de postgrado que integren ambas modalidades, puesto que dichos planes de formación profesional se ven enriquecidos 
con el instrumental metodológico y tecnológico, a la vez que diversifican la oferta académica, haciendo más atractiva la promoción de los programas de Maestría y Doctorado.

Finalmente, el estudiante que concluye los estudios superiores -habiendo usado cualquiera de las dos modalidades- es un profesional crítico y posee las herramientas teóricas y metodológicas que guiarán su labor profesional. Las modalidades tutelan tanto el proceso de formación como la metodología evaluativa, lo cual asegura el éxito integral de formación.

\section{CONCLUSIÓN}

Como se ha podido observar en la descripción de las modalidades en docencia, la concepción teórica es clara y establece diferencias que, de ser seguidas al pie de la letra, constituyen dos mundos completamente diferentes de abordar la labor docente en las aulas universitarias. Pero lo anterior no es tan cierto a la hora de llegar a los salones de clase, pues hay toda una tendencia a utilizar las fortalezas de cada modalidad en favor de la otra, tratando de maximizar la metodología y controlando las debilidades de cada una. Así, la DER del CIDE, establece que su modalidad es presencial y a distancia, pues entienden -desde sus orígenes- que ambas modalidades aportan beneficios al proceso de enseñanza-aprendizaje de los adultos.

Además, entra en juego el factor ético de los estudiantes, pues parte de la crítica que recibe la educación a distancia -especialmente la que se vale de recursos virtuales-, es que no hay un medio de evaluación transparente que asegure la veracidad del proceso de cada sujeto. Estos cuestionamientos me hacen pensar que los mismos argumentos se podrían exponer para confrontar la educación presencial, pues está en el estudiante mismo si a lo largo del 
proceso comete fraude o copia trabajos para presentarlos en el curso. Entonces, los argumentos que cuestionan el proceder ético en la modalidad a distancia son subjetivos en la medida en que cada estudiante es diferente y asume su responsabilidad conforme a sus principios e intereses personales.

Con respecto a la educación virtual, todavía faltan más estudios que describan los procesos educativos usando la virtualidad, pero los que abogan por esta modalidad educativa, arguyen que es una modalidad emergente (con características propias, mencionadas páginas atrás) e igualmente efectiva a la educación tradicional. Todavía faltan respuestas a cuestionamientos como si el aprendizaje mediado por computadoras mejora la capacidad de retención de los alumnos, ayuda a construir ideas a partir de bases simples, promueve nuevas perspectivas y horizontes conceptuales, etc.

En definitiva, hay quienes defienden la autonomía de las modalidades, aunque hay algunos docentes (con los que se ha hablado informalmente) que consideran que ambos abordajes son complementarios, y que se puede extraer de ellos aspectos positivos que colaboran en la estructuración y desarrollo de cursos, haciéndolos más interesantes e innovadores, siempre bajo las premisas de la reflexión académica, la auto-formación y la excelencia.

\section{REFERENCIAS BIBLIOGRÁFICAS}

Altbach, P. y McGill Peterson P. -editores- (2000). Educación superior en el siglo XXI. Desafío global y respuesta nacional. Editorial Biblos: Buenos Aires, Argentina.

Alvarado Céspedes, F. y otros (1999). Hacia la Construcción de un Sistema de Capacitación Judicial de Calidad. ILANUD: Sucre, Bolivia. 
Dengo, $M^{\text {a }}$ E. (2004). Educación costarricense. $9^{\text {na }}$ reimpresión. EUNED: San José, Costa Rica.

División de Educación Rural (2005). Plan de Estudios. Fotocopias.

Escotet, M.A. (1980). Tendencias de la educación superior a distancia. EUNED: San José, Costa Rica.

Flechsig KH. y Schiefelbein, E. (1985). Catálogo de Modelos didácticos. Versión 1985 - 1986, CIDE, Documentos de Trabajo, Santiago.

Henao Álvarez, O. (2004). "La enseñanza virtual en la educación superior". ICFES. En Web: http:// www.colegiovirtual.org/pr03pag.html

Tintaya, E. "Desafíos y retos de la educación virtual". En Web:

http://www.monografias.com/trabajos18/eduvirt/ eduvirt.tml

Muñoz Razo, C. "La universidad frente a los problemas y desafíos del siglo XXI". Recuperado el 20 de agosto de 2005 de : http://www.serprofessoruniversitario.pro.br/ ler.aspTEX488

Sarramona, J. (1994). Fundamentos de Educación. Grupo Editorial CEAC, S.A.: Barcelona, España.

Universidad Estatal a Distancia. Página Web. Recuperado el 20 de agosto de 2005 de: http://www.uned.ac.cr/

\section{CIBER-CONFERENCIA}

CON: Ramírez Sánchez, Iris Amalia, profesora de la Escuela de Educación, UNED.

\section{ENTREVISTA}

CON: Cerdas Rivera, Yadira, Sub-directora División de Educación Rural, CIDE. 